\title{
NOPT - New polishing techniques for scalable, light-weighted mirrors of different materials
}

\author{
Sebastiano Spinelli ${ }^{1}$, Luigina Arcangeli ${ }^{1}$, Ruben Mazzoleni ${ }^{1}$, Massimiliano Rossi ${ }^{1 *}$, Marco Terraneo ${ }^{1}$, Roman \\ Windpassinger ${ }^{2}$
}

${ }^{1}$ Media Lario Technologies, 23842 Bosisio Parini, Italy

${ }^{2}$ ESA , 23842 Bosisio Parini, Italy

\begin{abstract}
In the framework of an assessment of optical polishing techniques, ESA has signed a contract with Media Lario to deliver two $250 \mathrm{~mm}$ mirrors with a common optical design to be polished down to very tight surface requirements. NOPT mirrors are respectively made of Zerodur and AlSi alloy with electroless nickel and will they be polished by means of bonnet polishing. Mirrors are light-weighted up to $20 \mathrm{~kg} / \mathrm{m}^{2}$; the mechanical and optical design is proven to be scalable up to $1 \mathrm{~m} \mathrm{CA}$ surfaces. This paper reviews the mirrors opto-mechanical design, introduce the polishing and metrology strategy while highlighting the differences and the common point in fabricating such mirror in Zerodur and metal.
\end{abstract}

\section{New Optical Polishing Techniques project}

Modern polishing techniques for high performance optics often use of small area tools, in contrast to classical large area polishing techniques, where the complete optical surface to be polished or large part of is covered by the polishing tool, limiting the available surface shapes to plane or spherical ones. Using local area polishing techniques enables the polishing of aspherical and freeform surface shapes. In this framework ESA has signed a contract with Media Lario to deliver two $230 \mathrm{~mm}$ clear aperture (CA) mirrors polished down to very tight specifications (shown in Table 1) by means of Zeeko bonnet polishing process [1].

Table 1: NOPT mirror PSD requirements after polishing

\begin{tabular}{|c|c|}
\hline Requirement & $\begin{array}{c}\text { Integrated PSD value } \\
\text { (nm RMS) }\end{array}$ \\
\hline $\begin{array}{c}\text { Shape (Low Spatial } \\
\text { Frequency } \mathrm{f}<0.021 \mathrm{cy} / \mathrm{mm} \text { ) }\end{array}$ & 7.5 \\
\hline $\begin{array}{c}\text { Waviness (Mid Spatial } \\
\text { Frequency } \\
0.021 \mathrm{cy} / \mathrm{mm}<\mathrm{f}<1.1 \mathrm{cy} / \mathrm{mm})\end{array}$ & 3 \\
\hline $\begin{array}{c}\text { Roughness }(\mathrm{High} \mathrm{Spatial} \\
\text { Frequency } \mathrm{f}>1.1 \mathrm{cy} / \mathrm{mm})\end{array}$ & 0.7 \\
\hline
\end{tabular}

Mirrors will be light-weighted up to an areal density of $20 \mathrm{~kg} / \mathrm{m}^{2}$ and will be fabricated one in Zerodur and one in AlSi aluminium alloy coated with a polishing layer of electroless Nickel. The use of the AlSi alloy, that is CTE matched with the Nickel coating, allows the fabrication of an almost athermal design of the mirror as for the case of
Zerodur. The polishing process, the light weighting and mechanical design of the mirror are proven to be scalable up $1 \mathrm{~m} \mathrm{CA}[2]$.

\section{NOPT mirrors design}

Mirrors share the optical design adopted for the primary mirror of the ESA mission CHEOPS [3], apart the reduction of the CA from $330 \mathrm{~mm}$ to $230 \mathrm{~mm}$. This design was adopted in order to build a demonstrator known as being a suitable surface to be selected in space systems. The optical surface is thus hyperbolic, with a curvature radius of $742.756 \mathrm{~mm}$ and a conic constant $\mathrm{k}=-10.2456$.

While sharing the CHEOPS optical design, the mechanical design of the mirrors is completely different from the original one.

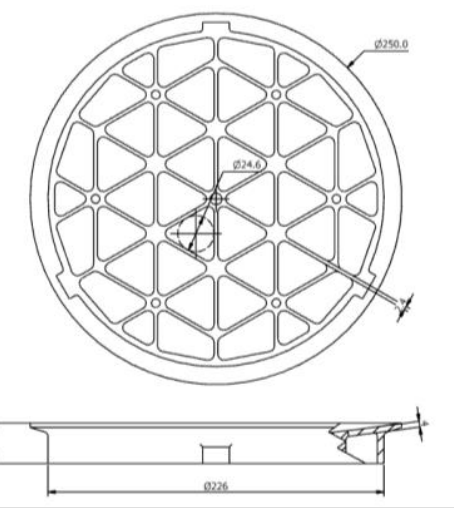

Figure 1: Skecth of the NOPT mirrors.

\footnotetext{
Corresponding author: massimiliano.rossi@medialario.com
} 
NOPT mirrors are designed to have an areal density of $20 \mathrm{~kg} / \mathrm{m}^{2}$ and yet a first eigenfrequency higher than 500 Hz. This has been achieved by designing a triangular shape modular pattern for the light-weighting scheme according to [4]. This approach fulfills also the requirement imposed by the ESA SoW of having a lightweighting scheme scalable up to $1 \mathrm{~m} \mathrm{CA}$.

\section{NOPT mirrors polishing}

Mirror polishing will be carried out by means of bonnet polishing machines. Media Lario has developed through the years different polishing processes specific both for Zerodur and AlSi electroless nickel plated mirrors able to bring down the surface errors to the requirements.

As a general description, the polishing process can be described as follows

I. Sub-surface damage removal: this is a polishing phase devoted to glass parts only in which material is uniformly removed from the surface in order to avoid superficial cracks induced by the grinding process [5]. Metal mirrors do not require this phase as they are diamond turned.

II. Low frequency errors (shape) are deterministically removed from the surface. In this polishing phase the bonnet transfer function is assumed to be point-like with respect to the shape error to be removed and is effective on LSF errors of Table 1

III. Mid-spatial frequency errors are deterministically removed from the surface by means of bonnet Precession polishing [6]: in this phase, the real bonnet transfer function is measured and deconvolved from the error map in order to obtain a more accurate dwell time map on the surface from the polishing tool to attack MSF of Table 1

IV. High frequency errors are stochastically removed lapping the surface with a proper bonnet-based process, in order to match the roughness HSF specification in Table 1.

As of now, the fabrication of both mirror substrates is completed. The Zerodur mirror polishing has just started, while the metal mirror diamond turning will be completed by the end of March. The project plan is to deliver both mirrors polished down to specifications before June 2019 .

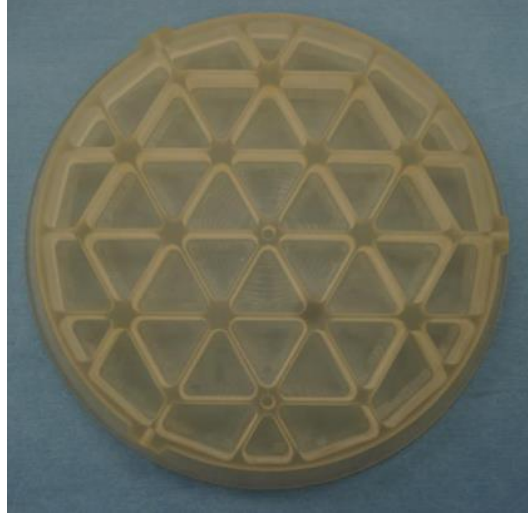

Figure 2: Zerodur NOPT mirror

\section{NOPT mirrors Metrology}

Media Lario has developed metrology tools to characterize the surface errors in the spatial frequency ranges indicated by mirror specifications.

Specifically, LSF and MSF will be addressed starting out from interferometric measurements with respect of a $\mathrm{CGH}$. A dedicated setup for having these measurements under the available 6" WYKO interferometer that minimizes the distortion induced by the mounting was designed with FEA and fabricated.

These measurements will provide the data for PSD definition up to 1.1 cycles $/ \mathrm{mm}$ frequency.

HSF will be measured by means of a Taylor Hobson CCI white light interferometer that has the capability to reconstruct the 2D surface PSD from $6 \mathrm{~mm}$ down to $1 \mu \mathrm{m}$ spatial wavelengths.

In order to discriminate the impact of the WLI optics in the PSD reconstruction, a correlation analysis between the WLI PSD and ARS measurements will be carried out on two 4" samples made of the same materials as NOPT mirrors. ARS was measured by means of the ALBATROSS apparatus, developed by Fraunhofer IOF Jena.

\section{References}

1. R. G. Bingham et al, "A novel automated process for aspheric surfaces,” Proc. SPIE 4093, 445-448, 2000

2. ESA ITT AO/1-8769/16/NL/RA, New Optical Polishing Techniques, Statement of Work, issue 1, 14/07/2016.

3. The CHEOPS team, "CHEOPS definition study report (Red Book)," ESA/SRE(2013), 2013.

4. D. Vukobratovich "Lightweight Mirror Design" in Optomechanical Engineering Handbook, CRC Press, 1999.

5. Lambropoulos, J. C., "From abrasive size to subsurface damage in grinding," OSA Technical Digest 8, 17-18, 2000

6. Walker et al., The 'Precessions' tooling for polishing and figuring flat, spherical and aspheric surfaces, Opt. Express 11, 958-964, 2003. 